\title{
REVISTA CIENTÍFICA
}

\section{Procesos de Gestión Tecnológica del Conocimiento EN Educación BáSICA}

\author{
Processes of Technological Management of Knowledge \\ IN BASIC EDUCATION
}

PhD. Fidel Parra, fparra@uisrael.edu.ec Universidad Tecnológica Israel, Quito, Ecuador.

Mg. Silvia Martínez, smartinez@uisrael.edu.ec Universidad Tecnológica Israel, Quito, Ecuador.

Mg. Henry Recalde, hrecalde@uisrael.edu.ec Universidad Tecnológica Israel, Quito, Ecuador.

Mg. Maryoribel Reañez, maryoribel20@gmail.com Universidad Tecnológica Israel, Quito, Ecuador.

Recepción: 15/11/2016 - Aceptación: 14/12/ 2016 - Publicado: 09/01/2017

\section{Resumen}

El propósito de esta investigación fue analizar los procesos de gestión tecnológica del conocimiento en educación básica, tomando como referentes teóricos a los autores Odreman (2014), Paniuagua y otros (2007), se utilizó un enfoque cualitativo interpretativo, mediante una revisión documental, los datos de las informaciones recabadas se interpretaron desde la óptica del saber; lógica y razón del investigador. Sobre esa base, los hallazgos evidencian resultados deficientes con respecto a los procesos de gestión tecnológica del conocimiento en las instituciones de Educación Básica de Venezuela donde se persigue la combinación del tratamiento de la información, con las capacidades de creatividad e innovación de los estudiantes, a través del uso de las Tecnologías de la Información y la Comunicación (TIC); por lo cual se realizan dos sugerencias concretas; la primera sugiere realizar una diagnosis en cada entidad educativa venezolana sobre las necesidades reales del nivel de educación básica con respecto a la sinergia entre el conocimiento y las TIC, para la inserción de sistemas y equipos de vanguardia. La segunda propone realizar reflexiones pertinentes que deben conducir a 
las comunidades de ese nivel educativo al manejo efectivo de las nuevas tendencias tecnológicas en educación, para coadyuvar a la pertinencia y calidad de la educación actual en Venezuela.

Palabras clave: Procesos, gestión tecnológica, conocimiento, educación básica, equipos, vanguardia.

\begin{abstract}
:
The purpose of this research was to analyze the processes of technological management of basic education, taking as theoretical references of the authors Odreman (2014) Paniuagua and others (2007), a qualitative interpretive approach was used, through a documentary review, the information collected was interpreted from the perspective of knowledge, logic and reason of the researcher. On this basis, the findings show deficient results with respect to the processes of technological knowledge management in the institutions of Basic Education of Venezuela where the combination of information processing is pursued, with the abilities of creativity and innovation of the students, through the use of Information and Communication Technologies (ICT); for which two concrete suggestions are made; the first one suggests making a diagnosis in each Venezuelan educational institution about the real needs of the basic education level with respect to the synergy between knowledge and ICT, for the insertion of state-ofthe-art systems and equipment. The second proposes to carry out pertinent reflections that should lead the communities of this educational level to the effective management of the new technological tendencies in education, to contribute to the relevance and quality of current education in Venezuela.
\end{abstract}

Keywords: Processes, technological management, knowledge, basic education, equipment, vanguard. 


\section{Ideas iniciales - Introducción}

La evolución de las organizaciones en el mundo ha exigido la asunción de una cultura que apunte al conocimiento, por ende, a la creatividad y la innovación que derivan de este. En razón de ello, si bien se requiere que los docentes cultiven nuevos patrones de pensamiento, para aprender en equipo, también se demanda en la actualidad que adapten sus actitudes a los cambios constantes del ambiente que los rodea, debiendo apegarse a las transformaciones sustanciales dadas con base en la tecnología.

Así se desprende de lo planteado por Rodríguez y Ochoa (2008), quien señala que no siempre se generan los resultados esperados, dado que son diversas las instituciones de educación básica que mantienen creencias y costumbres retrógradas, no se apegan a las transformaciones tecnológicas y nuevas tendencias educativas, lo que genera atraso y carencia de una gestión del conocimiento.

Por tal motivo, en las instituciones educativas se debe prestar atención, sobre todo en el nivel básico, dirigir las acciones pedagógicas y tecnológicas para la formación adecuada de los estudiantes. Estas acciones deben ir encaminadas al desarrollo de procesos que permitan su mejoramiento profesional y humano, hasta lograr un individuo competente.

Sin embargo, se ha observado en la educación básica venezolana, cierta resistencia al cambio que se produce ante las transformaciones del currículo básico, las nuevas estrategias y actividades académicas que deben derivar del enfoque constructivista en la educación, más aún, en la necesidad de la simplificación de procesos devenida del uso de las tecnologías, lo cual, en definitiva, desencadena consecuencias negativas en la calidad de la educación y en los procesos de aprendizaje de los estudiantes, como factor fundamental en los objetivos educacionales de la actualidad.

Cabe enfatizar que, en las instituciones educativas y más específicamente en el nivel básico, la gestión del director, de sus pares docentes y los colaboradores, debe orientarse hacia el cambio continuo; pero reconociendo la capacidad de aprendizaje y de innovación del conocimiento como una herramienta o recurso intangible imprescindible para las mismas, en la búsqueda de la consecución de las metas y objetivos.

Al respecto, Moreno (2005) señala a la gestión del conocimiento indispensable para el cambio en las ideas y acciones de los sujetos intervinientes en los procesos educativos, debiendo facilitar el capital intelectual y el desarrollo de mecanismos para la generación, procesamiento y uso de la información; que permitan la transformación de las instituciones y prepararlas para la formación de individuos idóneos para la sociedad actual.

Destaca de ese planteamiento, que el proceso de gestión del conocimiento involucra la capacitación, comprende la obtención de datos, los cuales al ser procesados son convertidos en información y al ser aplicados se convierten en conocimientos. Ante estas realidades surgen nuevos paradigmas en la educación basadas en la tecnología, la comunicación y el conocimiento, fundamentadas en la forma de representar, acceder y compartir el conocimiento en el siglo XXI es radicalmente diferente, con una tendencia a la transmisión del pensamiento inmediato y en forma de píldoras breves y significativas.

Es así que en algunas instituciones educativas que han sido observadas, pareciera evidenciarse algunas funciones críticas de la gestión en materia de desarrollo de conocimiento y de perfeccionamiento en tecnología, toda vez que sus actores, sobre todo, directivos y docentes, no integran la tecnología a las actividades administrativas o académicas de las organizaciones escolares.

De la misma manera, se observa de algunas manifestaciones de docentes en entrevistas informales suscitadas previas a este estudio, que en las planeaciones anuales no se atiende a la incorporación de tecnologías modernas, de avanzada, ni se desarrollan proyectos que permitan el aumento del nivel de conocimiento de los actores educativos a través de planes y programas interdisciplinares basados en las nuevas tecnologías. En tal sentido, no se potencia la innovación tecnológica, al no adquirir equipos nuevos, sino que se 
mantienen laborando con equipos obsoletos, lentos, que le imprimen complejidad a las tareas educativas administrativas y académicas. Por otra parte, no se visualiza a futuro una planeación tecnológica, para fijarse metas en cuanto a la adquisición y la asimilación de tecnología; no hay capacitación en el área para verificar las nuevas tendencias. Tampoco se atiende a problemas comunicacionales existentes en las instituciones educativas, desestimando nuevas vías para que los datos, la información y nuevos conocimientos generados, fluyan entre sus actores.

Finalmente, se ha observado negligencia y apatía para buscar la integración de los equipos de trabajo a través de dispositivos tecnológicos, ni se propicia la creatividad en los procesos educativos, por lo que se evidencia la necesidad de motivar al personal para convertirlos en entes significantes del proceso educativo, innovadores, que puedan, a través de su formación manejar centros y equipos de investigación y desarrollo.

Por otro lado, pareciera que el personal no ha asumido que es a través del conocimiento desde donde puede lograrse el máximo aprovechamiento de los recursos, tanto materiales técnicos y humanos, lo cual no siempre se ejercita de esa manera, muchas veces por falta de recursos, o por carencia de iniciativa del ente directivo o de los pares docentes, lo que decae en la ejecución de prácticas académicas y administrativas tradicionales, sin apego a la innovación cognoscitiva y tecnológica.

Con lo planteado, surge la inquietud del investigador en cuanto a analizar los procesos de gestión tecnológica del conocimiento en la educación básica venezolana. Ello, porque dentro de las organizaciones educativas es necesario esa gestión de aprender y compartir conocimiento para integrarlo a las actividades diarias, incorporando dicho proceso, en las rutinas directivas, académicas y administrativas. Con base en lo descrito en los párrafos precedentes, el problema objeto de estudio se formula de la siguiente manera: ¿Cómo se dan los procesos de gestión tecnológica del conocimiento en la educación básica venezolana?

Es por eso que como objetivo principal se pretende analizar los procesos de gestión tecnológica del conocimiento en educación básica venezolana. Por lo que se hace necesario conceptualizar la gestión del conocimiento buscando la inserción de la tecnología como elemento propio e identificando sus procesos en el nivel de educación básica, para de esta manera poder describir la implementación efectiva de las TIC en el escenario educativo del nivel básico venezolano a partir de sus funciones.

En tal sentido, se hace indispensable en la actualidad que las instituciones educativas del nivel básico conformen y ejerciten acciones transformadoras, que gestionen el conocimiento desde la perspectiva de la tecnología, adaptarse al cambio, influir en la comunicación efectiva, mejorar la toma de decisiones, pues no se puede permanecer aislado, se necesita innovación, lo que significaría un gran valor para elevar la misión y visión de las instituciones educativas venezolanas, manteniendo así a la organización unida como equipo de trabajo, garantizando que logre sus objetivos de una manera eficaz y eficiente.

Con base en tales referencias, este estudio se justifica desde el punto de vista social, en razón de que la comunidad educativa puede beneficiarse de las bondades de las TIC al ser implementadas en los diferentes espacios de las instituciones de educación básica; desde la perspectiva teórica se justifica, ya que se compilan informaciones relevantes sobre el tema; en el nivel práctico, porque sus resultados y conclusiones podrán ser puestos en práctica en las escuelas donde se ejecuten acciones pedagógicas propias del nivel básico; y desde el enfoque metodológico porque se utilizan métodos empíricos es decir, conocimiento adquirido por experiencia para su desarrollo.

\section{Fundamentos teóricos}

En este acápite se desarrolla información suficiente para el tratamiento de algunos puntos que relacionados con la temática en estudio representan un marco conceptual suficiente para dar explicación detalla- 
da a la temática expuesta, lo que significa recopilar el estado del arte de la misma, en aras de buscar el examen y la comprensión de lo expuesto. En tal sentido, se compendia información acerca de la gestión del conocimiento, en términos generales, la gestión tecnológica del conocimiento, la tecnología como elemento de la gestión del conocimiento, el proceso de la gestión tecnológica del conocimiento, y algunas estrategias de transferencia de tecnología, que aportan análisis al tema expuesto.

\section{Gestión del conocimiento}

La gestión del conocimiento se muestra como el proceso por el cual las organizaciones lo crean, almacenan y utilizan colectivamente. Este proceso de acuerdo a Piccoli (2005) incluye tres etapas: el aprendizaje organizacional, proceso por el cual se adquiere información; la producción de conocimiento, es el proceso de transformar e integrar la información en conocimiento utilizable; y la distribución del conocimiento, el proceso de diseminación del mismo a través de la organización.

Tal gestión del conocimiento, de acuerdo a Belly (2004), existe como práctica organizacional desde hace muchos años, en la época industrial se gestionaba lo tangible, en la era del conocimiento se gestiona lo intangible. De allí que pueda definirse como gestión estratégica de las capacidades intelectuales de los miembros de una organización, en este caso, educativa.

Asimismo, su fin resulta de añadir valor a los productos (la creación de un hombre apto a la sociedad en el caso del nivel educativo básico) y servicios (investigación, desarrollo extracurricular a través de la ejecución de actividades propias de los ejes transversales) que se ofrece en el contexto estudiantil para diferenciarlos competitivamente; e incluye todos los procesos relacionados con el desarrollo de experiencias y habilidades para aprender permanentemente y con autonomía que es la base fundamental de la escuela.

\section{Gestión tecnológica del conocimiento}

Tanto la gestión del conocimiento como la gestión de la tecnología buscan alcanzar las metas de las instituciones educativas mediante la obtención y administración del conocimiento y la tecnología que la institución requiere para ser competitiva. La tecnología es conocimiento aplicado, por tanto, ambas comparten actividades y principios.

Bajo esta perspectiva, según Malhotra (2001), la gestión tecnológica del conocimiento es el proceso que busca la sinérgica del tratamiento de los datos y la información mediante las capacidades de las tecnologías de la información y la comunicación (TIC) y las capacidades de creatividad e innovación de las personas. Así entonces, se define como el proceso tecnológico que aumenta la creatividad e innovación en la acción educativa.

Por su parte, Paniagua, López y Martín (2007), definen la gestión tecnológica del conocimiento como el conjunto de procesos y sistemas computacionales que permiten a una organización generar ventaja competitiva sostenible en el tiempo, mediante la gestión eficiente de su conocimiento, desarrollando las formas que se tienen que cumplir para la transformación del conocimiento desde la perspectiva de Nonaka (1991) que son: socialización, exteriorización, combinación e interiorización.

Para el caso de las instituciones educativas del nivel básico, la gestión tecnológica del conocimiento se debe adaptar a las exigencias, demandas y necesidades propias de cada institución. Es así como el Grupo de investigación en Interacción y e-learning de la Universidad de Salamanca y el Grupo de Excelencia de la Junta de Castilla y León, seleccionaron un proceso basado en los siguientes pilares, según García (2010): 
1. Gestión de la tecnología como parte del conocimiento del grupo.

2. Web 2.0

3. La integración de aplicaciones.

4. La Práctica del Conocimiento Abierto.

5. El uso de software libre.

En el mismo orden de ideas, la gestión del conocimiento se compone por un grupo de fases que se producen en forma cíclica, constituyendo un proceso como lo señala Probst, (citado por León, Ponjuan y Rodríguez, 2006), las cuales se denominan: identificación, adquisición, desarrollo, distribución, uso, retención y medición del conocimiento, siendo este un modelo que sirve para observar las etapas por las cuales debe transcurrir tal proceso de la gestión del conocimiento, a fin de que las organizaciones educativas se apeguen al cambio, a las transformaciones que la misma sociedad del conocimiento ha generado.

De manera similar, la gestión tecnológica del conocimiento debe entenderse según Zorrilla (2003) como un conjunto de métodos necesarios para el desarrollo de soluciones orientadas a generar las bases del conocimiento de valor para una organización educativa.

Cabe destacar que el proceso de gestión del conocimiento, en el caso de las organizaciones educativas del nivel básico en Venezuela, de acuerdo a lo que se infiere de los planteamientos del autor referido, se centra en el concepto de generación de valor asociado a la institución, valor representado por los recursos propios de la institución, a saber: el talento humano o capital intelectual o activo de conocimiento de la institución; además de los recursos materiales y financieros de los cuales disponga la entidad educativa de la cual se trate.

De allí que, con fines explicativos se desarrolla tal proceso en toda su amplitud en la socialización, creación, difusión y aplicación del conocimiento, aspectos que igualmente deben ser aplicados a la gestión tecnológica del conocimiento de las instituciones educativas en el nivel básico en Venezuela.

\section{Implementación efectiva de las TIC en el escenario educativo del nivel básico en Venezuela a partir de sus funciones}

Las Nuevas Tecnologías de la Información y Comunicación (TIC) "son aquellas herramientas computacionales e informáticas que procesan, almacenan, sintetizan, recuperan y presentan información representada de la más variada forma"; según Bracho (2006, p. 34), de lo cual es posible inferir que tales tecnologías se convierten en un conjunto de herramientas, soportes y canales para el tratamiento y acceso a la información. Constituyen nuevos soportes y canales para dar forma, registrar, almacenar y difundir contenidos informacionales, que pueden ser aplicados de manera eficaz en las instituciones educativas del nivel básico.

Igualmente, Castell (2004, p. 24) señala algunos ejemplos de las tecnologías como: la pizarra digital (ordenador personal + proyector multimedia), los blogs, el podcast y, por supuesto, la web; igualmente se destaca que son herramientas aplicadas a la educación que facilitan el aprendizaje, el desarrollo de habilidades y distintas formas de aprender, estilos y ritmos de los aprendices.

Con base en lo referido, se infiere que tales tecnologías son herramientas indispensables en la gestión educativa en todos sus ámbitos. De allí que el uso de las tecnologías de la información y la comunicación (TIC) debe incrementarse en la actualidad, debido a que estas ofrecen recursos y herramientas que facilitan el acceso a la información para realizar la gestión educativa, basados en la investigación y adecuación de los contenidos al entorno y su propia realidad. 


\section{Metodología de estudio/Materiales y métodos}

El presente estudio se enmarca en el paradigma cualitativo-interpretativo, desarrollando un tipo de investigación documental desde el análisis de la temática seleccionada, fundamentado en el criterio de Martínez (2010). Asimismo, con fines de recabar información, se atendió a la bibliografía recolectada de textos escritos, digitales, y algunas referencias electrónicas precisas y pertinentes. La información se procesa a través de la interpretación de los textos y documentos digitales recolectados, para examinarlos, analizarlos y lograr dar respuesta al objetivo de investigación formulado y dilucidar la temática seleccionada. El análisis de contenido, permitió examinar los referentes teóricos consultados, además de hacer uso de la interpretación del investigador, de su experiencia, lógica, y su real saber y entender, sobre los insumos teóricos y los referentes conceptuales hallazgos producidos.

\section{Análisis y discusión de resultados}

De las afirmaciones indicadas en los párrafos anteriores, puede inferirse que, en el caso de las instituciones educativas venezolanas, se debe atender al alumno a través del proceso de aprendizaje, pero debe ser visualizado desde la óptica digital, a fin de insertarse en la era de la información y del conocimiento de manera idónea.

Es decir que, en educación básica, esta formación debe incluir lo relativo a las nuevas tecnologías, como lo indica Reynolds (2003), lo que da paso entonces a la gestión tecnológica del conocimiento, porque la escuela ha tenido que insertarse a esta era de la información y del conocimiento no sólo por efectos de la globalización mundial, sino por las instrucciones de las autoridades educativas en cada país, tarea que incluye a todos los actores educativos, para la pertinencia, calidad educativa, y la perfección del producto generado: el alumno, quien pudiera aplicar su capacidad crítica con mayor intensidad a los servicios estudiantiles que recibe.

Pero, en las instituciones que son objeto de estudio cuando se pretende implementar la tecnología, algunos sujetos se resisten al cambio, tal vez debido a se ven en la necesidad de aprender a usar estos medios digitales, cuando hasta ahora se habían desenvuelto en un mundo analógico, sin pensar que la digitalización, facilitaría la competitividad de la institución educativa en todos los ámbitos, sobre todo, si se genera una plataforma tecnológica no solo para el nivel administrativo, sino para los alumnos (nativos digitales), quienes derivarán el manejo exitoso de programas informáticos capaces de transformar el entorno.

Esta innovación, entonces, en las instituciones educativas, debe derivar del uso de la tecnología, de nuevos equipos funcionales, de procedimientos más expeditos, dejando de lado procesos administrativos y educativos ortodoxos, que, en muchos casos restan simplicidad en las tareas y aumentan las horas hombre, lo que equivale mayores costos.

De lo especificado se evidencia que en las escuelas es necesario considerar un sistema de gestión de información, que facilite material actualizado sobre las necesidades de los usuarios con vistas a lograr una eficiente gestión del conocimiento. Con esa finalidad se deben crear plataformas de conocimientos, intranets, portales, escenarios, entre otras herramientas, con el objetivo de incentivar a los estudiantes del nivel básico, a empleados administrativos, docentes y directivos, a consumir e intercambiar información e incrementar su nivel cognitivo. Estos elementos deben manejarse por los directivos escolares con el objetivo de potenciar el proceso de gestión tecnológica de conocimiento.

Asimismo, el uso de las computadoras como elemento divulgador en la educación en el nivel básico, es una herramienta indispensable en la gestión administrativa que realiza el director, también en el caso de los docentes, se multiplican las posibilidades para la comunicación, que puede ir desde videoconferencia hasta el uso de correo electrónico como herramienta para facilitar la comunicación entre un grupo 
de trabajo (docentes-estudiantes). Con lo descrito, puede deducirse una de las principales ventajas que tiene una gestión tecnológica en la educación básica para los actores de las instituciones educativas investigadas; es el intercambio de información y comunicación entre ellos, haciendo más viable el proceso de conocimiento.

De lo descrito, es menester que el uso de las tic sea una meta a ser fijada por las instituciones educativas, en su planeación anual y cotidiana, sobre todo del nivel básico, en razón de ser este el escenario desde donde se forma la personalidad, la actitud, la conducta del estudiante que prontamente va a ingresar a su desempeño habitual, y a las escuelas universitarias donde así lo seleccionen para convertirse en el hombre del mañana.

\section{Consideraciones y reflexiones finales/Conclusiones y recomendaciones}

En la actualidad, las escuelas básicas en Venezuela requieren adecuarse a los cambios tecnológicos que se han venido suscitando en los espacios educativos y organizacionales en general, abriendo un compás a las nuevas tendencias, a nuevos equipos, al manejo de herramientas que simplifiquen los procesos y enrumben la organización hacia vías de comunicación e información, desde las cuales se divulgue el conocimiento tanto en la parte administrativa como en el área de la docencia.

De allí que en las organizaciones escolares en estudio se haya detectado la necesidad de contar con factores tecnológicos, que inducen a la interacción social, la cultura organizativa o el conocimiento tácito de los individuos, los cuales deben planificarse, preverse, aunque no superponiendo el uso único de la tecnología como factor de apoyo y éxito para la administración de la institución, sino también en los procesos áulicos. De la misma manera, no se puede dejar de promover planes conjuntos de aprendizaje tecnológico donde se implementen proyectos informáticos, o que el propio sujeto, por iniciativa personal, se sume a la innovación tecnológica para simplificar sus tareas, haciendo más agradable y más práctico su contexto dentro de la institución.

Es así que, en caso de que la organización carezca de un conocimiento específico necesario, debe buscarlo en su entorno para adquirirlo o simplemente desarrollarlo en su interior. En este sentido, deben producirse procesos de planeación de la formación, de la capacitación necesaria en los miembros del personal educativo, lo cual, propicia un mayor rendimiento, una mejor producción, y una productividad eficaz.

Cabe destacar a la vez que en ese nivel básico estudiantil resulta indispensable diferenciar la gestión tecnológica y la gestión del conocimiento, dado que los fundamentos de la gerencia del conocimiento toman en cuenta las condiciones que necesariamente deben darse para que el conocimiento se adquiera, se difunda, se utilice y se genere en la institución, basándose en el proceso de aprendizaje y la innovación. Estos principios frecuentemente se pasan por alto en la gestión de tecnología por creer que no son necesarios o por no entender su impacto en los resultados de la gestión.

Se requiere igualmente atender a la gestión de tecnología, pues se basa en la premisa de que los equipos tecnológicos se vuelven obsoletos y deben ser reemplazados, aumentando entonces la adquisición de máquinas modernas para la asimilación de los contenidos digitales. Lo cual debe ser así, porque los logros alcanzados hasta estos tiempos por la gestión de tecnologías han sido evidentes y se ven reflejados en la articulación de la estrategia organizacional y su desarrollo tecnológico. Esta inteligencia tecnológica es un campo emergente, que fluye para simplificar los procesos.

Es de hacer notar, que la inclusión de la tecnología en las instituciones de educación básica en Venezuela no se debe limitar solamente a la administración y facilidad en los procesos, sino que se debe incluir en ámbito pedagógico. En este punto es necesario aclarar que la inclusión de la tecnología en la formación estudiantil, no ha reflejado el resultado esperado (en donde se ha implementado), en opinión 
del investigador, debido a que se ha querido adaptar la pedagogía a los medios tecnológicos existentes, y no pensando en el establecimiento de una pedagogía tecnológica, que permita llevar a cabo una enseñanza eficaz, donde el docente solo sirva de orientador, guía y facilitador de ese proceso de aprendizaje.

En ese sentido, hoy se deben estructurar los espacios gerenciales, administrativos y académicos desde la gestión tecnológica del conocimiento, aplicando medidas innovadoras que aseguren la competitividad de la institución educativa y creando entornos virtuales de aprendizaje según la realidad de cada institución. Es inminente que, en el ámbito internacional los sistemas educativos de innovación influyen de una manera contundente en el desarrollo tecnológico de una nación, por tanto, se requiere orientar acciones presupuestarias y de capacitación para atender las tendencias globales, llevar la tecnología tanto a la administración como a la docencia en las instituciones educativas de nivel básico en Venezuela.

En tal sentido, cada institución educativa del nivel básico debe desarrollar y actualizar su estrategia de Innovación, y transferencia de tecnologías con la periodicidad pertinente según el dinamismo que le imprima su protagonismo y actuación en la globalización. Con ello se agilizan las mejores prácticas como una efectiva manera de transferir experiencias y conocimientos externos hasta la estrategia propia; debiendo buscar incluso alianzas estratégicas entre ellas para la mayor difusión del conocimiento, a fin de tratar de asimilar y mantener el ritmo de la innovación y desarrollo tecnológico globalizado. 


\section{Bibliografía}

Belly, P. (2004). El Shock del Management. (1era ed.). México. Editorial McGraw-Hill. Interamericana.

Bracho (2006). Tecnología de la información y su gestión. Una visión moderna de los Sistemas de información. (3era ed.) Colombia: Mc Graw Hill.

Castells, M (2004). Uso y difusión de las tecnologías del Internet para el acceso a la sociedad Red.

García, P. (2010). Gestión del conocimiento y de la tecnología. Selección de soluciones tecnológicas. GRupo de Investigación en interAcción y eLearning (GRIAL) Universidad de Salamanca. Disponible en http://grial.usal.es

León, M.; Ponjuán, G. y Rodríguez, M. (2006). Procesos estratégicos de la gestión del conocimiento. Acimed. Vol. 14 (2). Documento en línea. Disponible en: www.bvs,sld.cu/revistas/aci/vol14_2_06/aci08206.htm. Consulta: 20/12/2011.

Malhotra, Y. (2001). Gestión del conocimiento y modelo comercial.Innovación. Universidad de Murcia. España. Idea Group Publishing.

Martínez M., M. (2010). Investigación cualitativa. México: Editorial Trillas.

Moreno (2005). Administración de empresas. México; Editorial Limusa.

Nonaka, I. (1991). Knowledge-Creating Company. Harvard Business Review; Nov/Dec91, Vol. 69 Issue 6, p96, 9p, 3.

Odreman R., (2014). Gestión tecnológica: estrategias de innovación y transferencia de tecnología en la industria. Revista Universidad, Ciencia y tecnología. Volumen 18, No. 73, Venezuela, Puerto Ordaz.

Paniagua A., López A., y Martín R. (2007). Fundamentos de la gestión tecnológica del conocimiento. España: Murcia, Universidad de Murcia, Revista Editum, Servicio de Publicaciones 2007.

Piccoli (2005). Administración del talento humano, como factor gestionador de la productividad individual, visionada en el marco de la cultura de las organizaciones integradoras en el Sur de Nápoli - Italia. Talento y Cultura Organizacional. Editorial Trillas. Colombia.

Reynolds (2003). Sistemas de Información. México: Ediciones Trillas.

Rodríguez P., Ochoa M., (2008) La cultura organizacional en el enfoque transdisciplinar de la gestión tecnológica ambiental. Revista ACIMED, La Habana, Cuba. Disponible en http://scielo.sld.cu/scielo.php?script=sci_arttext\&pid=S1024-94352008000 800003.

Zorrilla, F (2003). La Gerencia del Conocimiento y la Gestión Tecnológica. Colombia: Editorial Universidad de Los Andes. 